\title{
Capillary Microsampling of Mouse Blood in Early Pre-Clinical Studies: A Preferred Alternative to Dried Blood Spot Sampling
}

Jennie Roberts*, Ian Wilson, Alan T Henley, Meirion Richards, Ching Thai and Florence I Raynaud

Cancer Research UK Cancer Therapeutics Unit, The Institute of Cancer Research, 123 Old Brompton Road, London, SW7 3RP, UK

\begin{abstract}
Recent advances in sensitivity of analytical instrumentation has resulted in the routine practice of low-volume blood sampling in pre-clinical pharmacokinetic studies. Mice, the species of choice in oncology, have a limited blood volume; hence demand a small volume sampling strategy to avoid unwanted physiological side effects. When assessing drug exposure in mice, a serial sampling methodology requires less than $20 \mu$ of blood per time point in order to comply with animal welfare guidelines.
\end{abstract}

Various microsampling techniques have been employed in pharmacokinetic studies to achieve exact sampling of low blood volumes. The aim of this work was to explore capillary microsampling as an alternative methodology to dried blood spot sampling for the analysis of whole blood in mouse pharmacokinetic studies. Concentration-time profiles of both blood sampling techniques were compared following intravenous administration of four compounds to female Balb C mice. In each case pharmacokinetic parameters from the two different sampling methods were equivalent.

Compared to dried blood spots, capillary microsampling provided an inexpensive, reliable and robust method for low volume sampling in the pre-clinical pharmacokinetic setting. In vivo and bioanalytical workflows were integrated with the result of improving laboratory efficiency and lowering consumable costs. Compared to the previous blood spot method there was an improvement in animal welfare through faster handling times due to refined microsampling procedures.

Keywords: Capillary micosampling; Dried blood spots; Pharmacokinetics

\section{Introduction}

Pre-clinical pharmacokinetic (PK) studies establish the key parameters of clearance, volume of distribution and oral bioavailability for novel chemical entities in drug discovery. Following favourable in vitro screens for metabolism and permeability, early in vivo evaluation ensures that chemical series and potential drug candidates with poor in vivo profiles are deprioritised early, focusing project resources on chemical series and lead compounds with favourable pharmacokinetics. The selection of pre-clinical species may vary; the rat is often used in early PK studies. However, mice are preferred as disease models of oncology; either as genetically engineered animals or as athymic mice bearing human tumour xenografts $[1,2]$.

Using mice in routine PK studies can be challenging as total blood volume, often less than $1.5 \mathrm{ml}$, limits the size and number of samples that can be collected. In the past, the poor sensitivity of mass spectrometers required sample concentration of large volumes of plasma and discrete sampling strategy was then required to generate concentration-time profiles for a full PK, with an individual mouse used for each time point, minimising the physiological effect of taking large blood volumes. This approach has an inherent disadvantage as many individuals are used to create one concentration - time profile, generating average values with inter-animal variability and dosing error.

Direct analysis of blood instead of plasma has the advantage of being directly related to physiologically parameters, such as blood flow, used in the interpretation PK data.

Serial bleeding, an alternative method of sampling, provides a complete concentration-time profile of a compound by sampling just one animal over the time course. Large volume sampling cannot be applied to a full pharmacokinetic time course in mice as the volume of blood required would exceed animal welfare guidelines. Applying a low volume sampling strategy to serial bleeding has the advantage of providing an estimation of inter-animal variability throughout the time course as well as reducing study costs through a significant reduction in mice [3]. Introducing serial sampling has the benefit of conforming to the 3Rs (reduce, refine, and replace), an obligation for every project license under the Animals Scientific Procedures act 1986 [4]. A typical pharmacokinetic study using eight time points per dosing route (intravenous and oral drug administration with vehicle controls) would require sixty-four mice for traditional large volume plasma samples, whereas a blood microsampling methodology would use only eight.

A number of different methods for low volume sampling of matrix have been utilised, a popular one in recent years being the use of dried blood spots (DBS). Blood is collected and spotted onto filter paper and allowed to dry prior to punching disks of equal size for analysis. This technique has gained acceptance across many laboratories due to the ease of use, storage and extraction [5-7]. For the majority of analytes there is no requirement for centrifugation or storage on ice during the sample collection process and the cards can be stored at room temperature and can be shipped easily. The critical assumption in using DBS lies with sample homogeneity on the card or paper, and it is known that haematocrit values (a measure of the cell volume in blood) can

*Corresponding authors: Roberts J, The Institute of Cancer Research, Division of Cancer Therapeutics, Cancer Research UK Cancer Therapeutics Unit, The Institute of Cancer Research, 15 Cotswold Road Sutton, Surrey SM25NG, UK, Tel: 44(0)2087224212; E-mail: Florence.raynaud@icr.ac.uk

Received April 13, 2016; Accepted April 22, 2016; Published April 29, 2016

Citation: Roberts J, Wilson I, Henley AT, Richards M, Thai C, et al. (2016) Capillary Microsampling of Mouse Blood in Early Pre-Clinical Studies: A Preferred Alternative to Dried Blood Spot Sampling. J Bioanal Biomed 8: 028-033. doi:10.4172/1948593X.1000149

Copyright: $@ 2016$ Roberts J, et al. This is an open-access article distributed under the terms of the Creative Commons Attribution License, which permits unrestricted use, distribution, and reproduction in any medium, provided the original author and source are credited. 
impact the quality of the data [8]. Blood spot size varies with viscosity so a higher haematocrit value will reduce the spread of the blood on the card, a disk of the same size can therefore contain variable amounts of matrix and analyte. Confidence in reproducible analyte recovery is therefore contingent on even distribution of blood on the cards or on the punching of the whole spot which can vary in size. Capillary microsampling (CMS) is a relatively new technique, championed by Ove Jonsson [9]. Samples are collected and stored in capillaries prior to extraction. The extraction process involves addition of a washout liquid to the capillaries, protein precipitation of the capillary washout, and finally centrifugation to yield supernatant for LC-MS/MS analysis.

The primary aim of this investigation was to compare the PK results obtained from two distinct sampling methods: dried blood spot sampling which we have routinely used in our laboratory for over five years and capillary microsampling which we are testing in this study. A secondary aim was to integrate the in vivo and bioanalytical workflows, with a specific focus on the use of automated liquid handling systems. Small molecules (MW range: 341.8-569.6 Da) were chosen from multiple pre-clinical projects based on diverse physiochemical and pharmacokinetic properties (Table 1). Overall methodology and results in the form of PK profiles and parameters were compared for each sampling method.

\section{Materials and Method}

\section{Chemical and solutions}

UPLC/MS grade water, formic acid and methanol were obtained from Biosolve, France. Dimethyl sulfoxide (DMSO), saline and Tween 20 were purchased from Sigma Aldrich, UK. Test compounds ICR1, ICR2 and ICR3 and ICR4 were synthesised at The Institute of Cancer Research (ICR, London, UK). Molecular weight and physicochemical properties are presented in Table 1.

\section{Materials}

Heparinised glass capillary tubes ( $10 \mathrm{ul}, 30-32 \mathrm{~mm}$ minicaps $\left.{ }^{\circledR}\right)$ with exact end-to-end volume were purchased from Hirschmann (Germany) and Jaytec (UK). Plates (96 deepwell, Fisherbrand, UK) used for sample collection. Whatman FTA DMPK-B DBS cards (GE Healthcare, UK) were used together with $6 \mathrm{~mm}$ Uni-Core ${ }^{\text {max }}$ punches and cutting mats from Harris (GE healthcare, UK). Gilson MICROMAN ${ }^{\circledR}$ positivedisplacement pipettes were used to transfer blood onto DBS cards.

\section{In vivo PK studies}

All in vivo studies were performed in accordance with UK Home Office regulations, ICR ethical review processes and Cancer Research Institute guidelines [10].

Due to volume collection limitations, sufficient blood could not be obtained from one animal for both sampling methods, therefore two separate groups of animals were used. Female BALB/C mice, weighing 18-20 g, were obtained from Charles River Laboratories (Margate, Kent) and acclimatised for a minimum of 5 days prior to use. Each experiment required four mice for each sampling method (one control, three drug treated). Compounds were dissolved in DMSO and prepared for dosing by addition of saline and tween 20. Mice were warmed at $37^{\circ} \mathrm{C}$ for ten minutes in a Datesand mini-thermacage prior to dosing. Following intravenous administration, via the lateral tail vein, samples were collected by bleeding from the opposing vein to the dosage site at $5,15,30 \mathrm{~min}$ and $1,2,4,6$ and $24 \mathrm{~h}$.

\section{Sample collection}

CMS samples were taken using capillary tubes $(10 \mu \mathrm{l})$ and snap frozen by placing in a $2 \mathrm{ml} 96$ deep well plate on dry ice. The plate was sealed after sample collection and stored at $-80^{\circ} \mathrm{C}$. It is essential that the samples are frozen prior to extraction as this reduces possible non-specific binding of the analyte to capillaries and plates. Samples extracted without a freeze-thaw cycle did not produce reliable, linear calibration lines (data not shown).

DBS samples were collected via Jaytec capillaries (exact volume $20 \mu \mathrm{l})$ and spotted onto Whatman B DBS cards using a capillary tube pipette to dispense the $20 \mathrm{ul}$ of blood.

\section{Analytical stock preparation}

Separate weightings were used to prepare two stock solutions for each compound ( $1 \mathrm{mM}$ DMSO). Liquid handling automation (Hamilton Star, Switzerland) was used to produce working solutions for quality controls and calibration standards in DMSO. The top five standard concentrations were prepared by spiking directly from the stock solution into a well plate with DMSO. These working solutions were serially diluted to produce the lower standard concentrations. Similarly, quality control solutions were prepared from stock solutions and serial dilutions with DMSO. All DMSO stock solutions were stored at ambient conditions.

\section{Calibration and quality control preparation}

Fresh tail vein blood, pooled from two un-treated female BALB/C mice, was used to prepare eight calibration standards ( $1 \mathrm{nM}$ to 25 $\mu \mathrm{M})$ and four quality control (QC) samples (12.5 $\mathrm{nM}$ to $3750 \mathrm{nM}$ ) by manually spiking DMSO working solution $(2 \mu \mathrm{l})$ into blank blood $(40 \mu \mathrm{l})$ and mixing gently before spotting on DBS cards $(20 \mu \mathrm{l})$ and for CMS collection $(10 \mu \mathrm{l})$. The calibration standards and QCs for both methods were prepared on the day of study to allow for DBS cards to dry overnight in ambient conditions and for capillaries to be stored alongside samples at $-80^{\circ} \mathrm{C}$.

Capillary samples were defrosted and washout solution $(125 \mu \mathrm{l}, 30 \%$ $\mathrm{MeOH}: 70 \% \mathrm{H}_{2} \mathrm{O}$ ) was added to each plate well using the liquid handling automation. Washout volume may be varied; enough is required to wash the blood out of the capillary and give a homogenous blood-water mix, however, over dilution may be problematic for detection on some analytical platforms. In this method $125 \mu$ l was deemed to be sufficient to provide a homogenous mix with enough volume to permit a second, lower volume extraction if required.

Washout composition can also be varied to suit specific analytes. After testing over forty compounds with a range of AlogP values

\begin{tabular}{|c|c|c|c|c|c|c|c|}
\hline \multirow{2}{*}{ Compound } & \multirow{2}{*}{ MW } & \multirow{2}{*}{$A \log P$} & \multirow{2}{*}{ Ionisation State } & Measured & \multirow{2}{*}{ TPSA } & \multirow{2}{*}{ H Bond Donors } & \multirow{2}{*}{ H Bond Acceptors } \\
\hline & & & & $\log D$ & & & \\
\hline ICR1 & 446.6 & 4.231 & weak base & 4.53 & 102.66 & 2 & 9 \\
\hline ICR2 & 410.9 & 2.352 & neutral & 1.14 & 89.07 & 3 & 7 \\
\hline ICR3 & 569.6 & 4.171 & strong base & 1.73 & 96.03 & 2 & 9 \\
\hline ICR4 & 341.8 & 2.193 & strong base & 1.43 & 70.83 & 3 & 5 \\
\hline
\end{tabular}

Table 1: Four compounds from different pre-clinical projects were chosen on the basis of their molecular weight, chemical properties and PK profiles. 
Citation: Roberts J, Wilson I, Henley AT, Richards M, Thai C, et al. (2016) Capillary Microsampling of Mouse Blood in Early Pre-Clinical Studies: A Preferred Alternative to Dried Blood Spot Sampling. J Bioanal Biomed 8: 028-033. doi:10.4172/1948-593X.1000149

it was determined that some organic solvent, such as methanol or acetronitrile, is necessary in the washout as this will help solubilise and stabilise the more lipophilic compounds. Too much organic will cause protein precipitation in the capillaries and should be avoided. A washout content of $30 \%$ organic was shown to be optimal for the analytes under investigation. The overall process of collection and processing of the CMS is described in Figure 1.

Plates were sealed and thoroughly mixed, particularly by hand in the longitudinal direction of the capillaries, as this is required to fully wash out the blood. Mixing was considered complete when the bloodwater mix was homogenous. An aliquot of $75 \mu$ l blood - washout mix was transferred to another deep well plate, again using automated liquid handling, and protein precipitation was performed using a 1:3 ratio of methanol $(225 \mu \mathrm{l})$ (containing internal standard at $500 \mathrm{nM})$, aliquoted by automation.

DBS cards were punched into $6 \mathrm{~mm}$ disks centred on the blood aliquots and transferred to a deep-well plate and extracted with $200 \mu \mathrm{l}$ methanol containing internal standard at $500 \mathrm{nM}$.

Plates were mixed and centrifuged for $30 \mathrm{~min}$ at $1800 \mathrm{~g}$. Supernatant $(50 \mu \mathrm{l})$ was diluted with $\mathrm{MeOH} / \mathrm{H}_{2} \mathrm{O}$ (50 ul) ready for LC-MS/MS analysis.

\section{Recovery following CMS and DBS extraction}

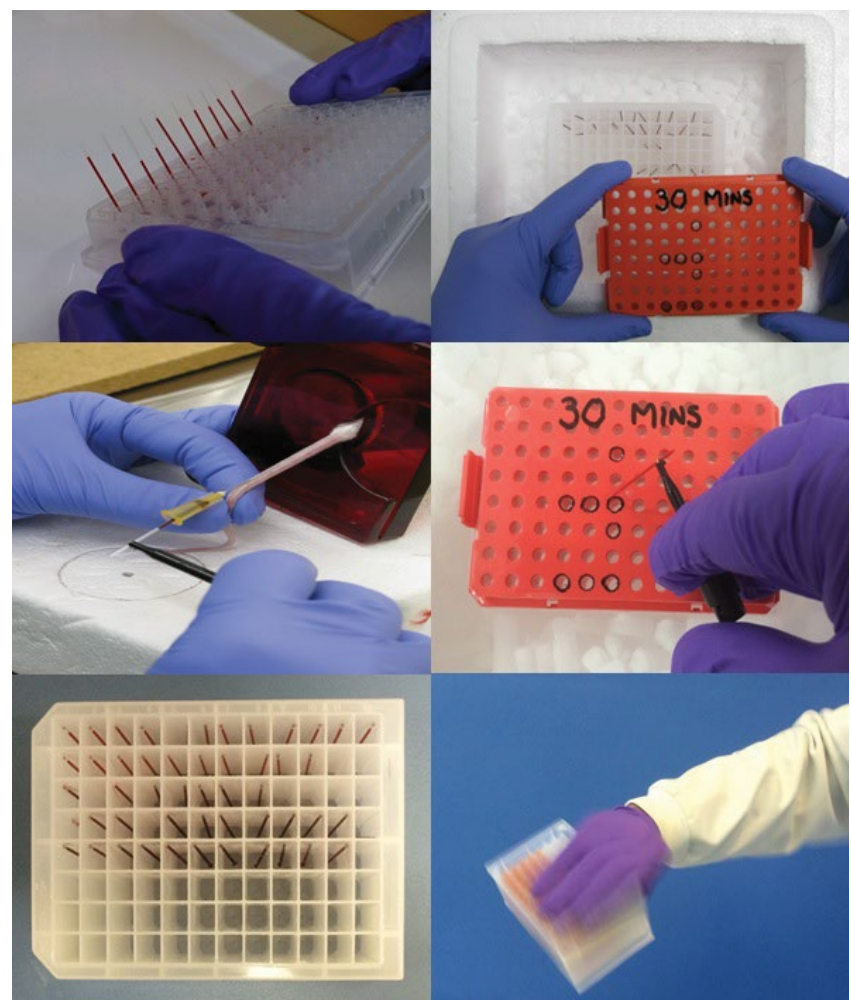

Figure 1: Illustration of the workflow used to carry out a pharmacokinetic study using CMS. Photos left to right top to bottom: (A) Calibration standards are prepared in a 96 well plate at the time of the in vivo experiment. Capillary action wicks the blood up the capillary tube. (B) Samples are stored on dry ice over the time course of the in vivo experiment. (C) Mouse bled via tail venepuncture, blood is collected into a capillary. (D) Templates are used to help in vivo technicians place samples in the correct wells. (E) Samples are thawed ready for extraction by the bioanalytical laboratory. (F) Following addition of the washout solvent, the plates are sealed and shaken in the longitudinal direction.
Control extracts were spiked with compound and compared to extracted samples for both sampling methods.

LC-MS analysis: Separations were performed on Waters Acquity LC system controlled through Analyst software. Samples were injected $(8 \mu \mathrm{l})$ on a Phenomenex Kinetex C18 column $2.6 \mathrm{u}\left(50^{*} 2.1 \mathrm{~mm}\right) 100$ $\AA$ with a Krudcatcher column guard maintained at $45^{\circ} \mathrm{C}$. The flow rate was $0.6 \mathrm{ml} / \mathrm{min}$ and a six minute gradient used mobile phase $\mathrm{A}(0.1 \%$ formic acid) and $\mathrm{B}(\mathrm{MeOH})(95 / 5$ to $0 / 100$ linear over 4 min $0 / 100$ to 95/5 from 5-5.2 min). An ABSciex QTRAP4000 triple quadrupole mass spectrometer was used in positive mode with an APCI source $\left(450^{\circ} \mathrm{C}\right.$ curtain gas $\left.35 \mathrm{psi}\right)$. Multiple reaction monitoring was used to detect the analyte and internal standard Table 2 for MS parameters. APCI was preferred over ESI to avoid potential ion suppression from the dosing vehicle surfactant. Standard curves were run in singlicate at the beginning of the run and quality controls in duplicate at the end of the curve and at the end of the analytical run.

\section{Acceptance criteria for analytical runs}

The calibration lines were analysed by linear regression and optimal weighting. No analytical validation was carried out for these studies. Concentrations were deemed acceptable if the nominal value of the analyte was within $15 \%$ of nominal concentration and $20 \%$ for the lower limit of quantification.

Six out of 8 quality controls had to be within $15 \%$ of nominal values at least one at each level.

\section{Pharmacokinetic data analysis}

Phoenix WinNonLin (Pharsight, USA) was used to calculate area under concentration-time curves $\left(\mathrm{AUC}_{\text {last }}\right)$ in order to determine $\mathrm{PK}$ parameters of clearance, half-life and volume of distribution.

\section{Determination of $\log$ D7.4 values}

Log $\mathrm{D}_{74}$ values were determined via an in-house developed HPLC method.

Calibration was achieved by comparing the retention time of eight commercially available drugs with a range of Log D7.4 between -1.38 and 5.5, and correlating these retention times against literature Log D7.4 values of the compounds. The calibration was validated by comparing HPLC-determined Log D7.4 values of two other commerciallyavailable drugs with literature Log D7.4 values.

The HPLC Log D7.4 values of in-house compounds were determined by substituting the compounds' retention times into the equation obtained from the linear part of the calibration curve.

\section{Result}

The recoveries from CMS and DBS extracts were complete for ICR2 and ICR3 while ICR1 and ICR4 were recovered better from CMS and DBS extracts respectively (Table 3 ). The recovery for ICR1 and ICR4

\begin{tabular}{|c|c|c|c|c|}
\hline \multirow{2}{*}{ Compound } & \multicolumn{2}{|c|}{ MRM transition $(\mathbf{m} / \mathbf{z})$} & DP & CE \\
\cline { 2 - 5 } & Precursor & Product & & \\
\hline ICR1 & 447.2 & 377.1 & 66 & 41 \\
\hline ICR2 & 411.3 & 375.1 & 91 & 41 \\
\hline ICR3 & 570.3 & 127.2 & 86 & 47 \\
\hline ICR4 & 342.2 & 147.1 & 41 & 39 \\
\hline Internal Standard & 299.2 & 177.0 & 80 & 30 \\
\hline
\end{tabular}

Table 2: Analyte MS/MS parameters for QTRAP4000 in ESI: Following tuning with compound optimiser, one transition was selected on the basis of sensitivity and selectivity in matrix. 
were above $50 \%$ and consistent across the concentration range which was considered acceptable for these early studies given the fact that standard curves quality controls and samples were extracted from blood. No signal was detected in any of the control samples from animals treated with vehicle alone (data not shown). The range of quantification varied between compounds with saturation observed at $25000 \mathrm{nM}$ for all compounds but ICR1 in the CMS assay and ICR4 in the DBS assay. The quality controls in Table 4 demonstrate that the analytical runs were within the acceptance criteria set for these early studies.

Despite the differences in recovery, sensitivity and dynamic range following both sampling methods, the average concentration-time profiles for each of the four compounds were superimposable for all analytes (Figure 2).The current sensitivity of our LC-MS/MS systems was sufficient to obtain meaningful PK profiles for early evaluation of novel chemical entities.

Systemic exposure (AUC) was used to calculate pharmacokinetic parameters of blood clearance, half-life and volume of distribution (Table 5). There was no significant difference between AUCs, half-lives and volume of distributions derived from CMS and DBS data (MannWhitney test $\mathrm{P}<0.05$ ). The variation in exposure was less than $5 \%$ for 3 compounds and $16 \%$ for ICR3. Variations in volume of distribution were more pronounced but less than 30\% difference was observed between the two sampling methods often mirroring variation within a single sampling method. This was therefore considered acceptable in these early PK studies.

We concluded that concentration-time profiles and PK data generated from our CMS and DBS studies were comparable for the investigated compounds in-vivo. The full sample plates were delivered to the bioanalytical laboratory where automated liquid handling was used to add washout solution and to protein precipitate the samples ready for LC-MS/MS analysis, simplifying the analytical workflow. The use of laboratory automation had the benefits of increasing precision, saving analyst time and improving data quality. Although automation of DBS is possible with the purchase dedicated systems or LC-MS/MS add-ons, the equipment required is not always compatible with the layout of existing multipurpose liquid handling systems.

\begin{tabular}{|c|c|c|c|c|c|}
\hline & & ICR1 & ICR2 & ICR3 & ICR4 \\
\hline \multirow{2}{*}{$\begin{array}{c}\text { Recovery } \\
(\%)\end{array}$} & CMS & $100 \pm 1$ & $54 \pm 2$ & $100 \pm 5$ & $59 \pm 1$ \\
\cline { 2 - 6 } & DBS & $89 \pm 6$ & $59 \pm 3$ & $100 \pm 3$ & $69 \pm 3$ \\
\hline
\end{tabular}

Table 3: Percentage recovery: To test recoveries we spiked control extracts from CMS and DBS with $1 \mu \mathrm{M}$ of compound and compared the results with a $1 \mu \mathrm{M}$ CMS and DBS extracted sample for each compound.

\begin{tabular}{|c|c|c|c|c|c|}
\hline \multicolumn{2}{|c|}{$\begin{array}{c}\text { Quality } \\
\text { Controls }\end{array}$} & ICR1 & ICR2 & ICR3 & ICR4 \\
\hline \multirow{2}{*}{$12.5 \mathrm{nM}$} & $\mathrm{CMS}$ & $12.5 ; 15.6$ & $14.3 ; 13.3$ & $13.9: 15.1$ & $13.7 ; 13.6$ \\
\cline { 2 - 6 } & DBS & $13.0 ; 16.2$ & $12.8 ; 15.8$ & $12.8 ; 13.8$ & $14.8 ; 11.2$ \\
\hline \multirow{2}{*}{$125 \mathrm{nM}$} & $\mathrm{CMS}$ & $113 ; 119$ & $137 ; 149$ & $135 ; 115$ & $107 ; 116$ \\
\cline { 2 - 6 } & DBS & $113 ; 118$ & $140 ; 126$ & $119 ; 108$ & $123 ; 114$ \\
\hline \multirow{2}{*}{$1250 \mathrm{nM}$} & $\mathrm{CMS}$ & $1080 ; 1190$ & $1400 ; 1130$ & $1168 ; 1341$ & $1090 ; 1110$ \\
\cline { 2 - 6 } & DBS & $1290 ; 1177$ & $1230 ; 1420$ & $1188 ; 1201$ & $1190 ; 1270$ \\
\hline \multirow{2}{*}{$3750 \mathrm{nM}$} & CMS & $3707 ; 3666$ & $4290 ; 3910$ & $3545 ; 3265$ & $3350 ; 3590$ \\
\cline { 2 - 6 } & DBS & $3708 ; 4121$ & $3950 ; 3610$ & $3545 ; 3265$ & $3710 ; 3710$ \\
\hline
\end{tabular}

Table 4: Quality controls for both methods: A comparision of QC concentrations
The transition from DBS to capillary microsampling required minimal training as sample collection, handling and extraction are very simple. Sampling templates, guiding correct placement of samples into 96 well plates, were created in order to facilitate an integrated in vivo analytical method. Different storage conditions for CMS compared to DBS had a significant impact in the use of laboratory storage space. DBS cards were stored at ambient temperature in racks with a full PK having a similar footprint to an A4 piece of paper. In contrast, CMS samples were stored in freezers $\left(-80^{\circ} \mathrm{C}\right)$ in the 96 deepwell plate format, often just for $24 \mathrm{~h}$, until extraction. This maintained a clear and tidy lab space and protected samples from contamination and damage.

Blood sampling using CMS was speedy and efficient compared to the DBS method. In part this was due to the reduced quantity of blood collected, reducing the time taken to fill the sample capillary with no additional card spotting. Using smaller volumes also raised the possibility of adding additional time points into the study, either repeating time points or lengthening the study.

Care must be taken to avoid transferring other contaminants when spotting blood onto DBS cards, particularly in a busy laboratory setting where dosing solutions may be present. In contrast, capillaries are sampled and immediately placed in a 96 well plate away from sources of contamination.

Extracting blood directly from capillaries eliminates haematocrit issues experienced with DBS. While it may be possible to take the entire blood spot, in order to avoid a potential missampling due to compounds modifying the haematocrit, it is often not practicable to do so with the circular punches. Spots may not fit into the disk shape and cutting out the whole spots would be time consuming, particularly when compared to the time taken to process samples with our current CMS workflow. Current LC-MS technologies should be sufficiently sensitive to detect nanomolar levels of analytes in complex matrices like blood. However, blood volumes used in this CMS method can pose an analytical challenge as smaller samples and dilution with washout will reduce analyte concentrations in comparison to the DBS samples.

A benefit of introducing smaller sampling volumes, while only a reduction from $20 \mu \mathrm{l}$ to $10 \mu \mathrm{l}$, is the shortening of time spent bleeding animals. This reduces stressful handling and is considered a refinement of the technique by providing welfare benefits through reduction in restraint time. This reduction in volume could potentially be applied to the DBS method although manufacturers of the cards that we use recommend 15-20 $\mu$ per spot. Perceived limitations in DBS have led to a renewed interest in liquid microsampling using capillaries $[11,12]$.

There is a significant financial advantage to using CMS over DBS methodology.

CMS sampling uses one capillary for sampling and storage, whereas DBS requires both a larger sampling capillary and DBS card. The cards have space to spot four samples but are relatively expensive in comparison to heparinized capillaries.

Replacing the use of DBS cards by using the CMS methodology therefore results in a significant decrease in consumable costs; capillaries for a full CMS PK cost seven-fold less than a set of DBS cards and sampling capillaries. Overall, a seven-fold reduction in costs is achieved by eliminating DBS in favour of CMS. While CMS samples require freezing this does not incur any additional cost as both in vivo and bioanalytical laboratories have freezers and very little space is needed to store the sample plate. Although this study used 
Citation: Roberts J, Wilson I, Henley AT, Richards M, Thai C, et al. (2016) Capillary Microsampling of Mouse Blood in Early Pre-Clinical Studies: A Preferred Alternative to Dried Blood Spot Sampling. J Bioanal Biomed 8: 028-033. doi:10.4172/1948-593X.1000149
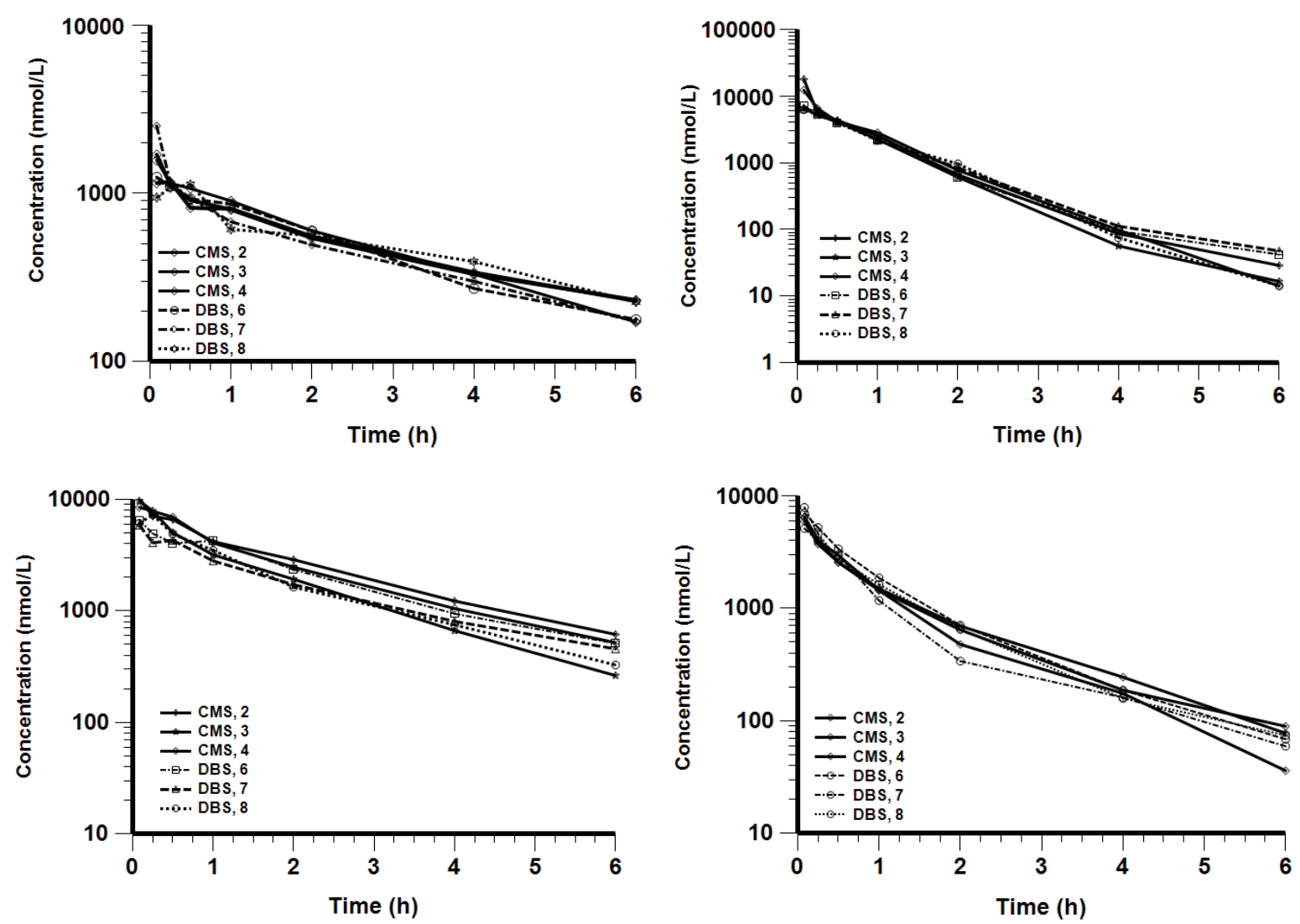

Figure 2: PK profiles after intravenous dosing with bolded lines representing CMS sampling and dotted lines DBS sampling. Individual mice are numbered; CMS 2, 3,4 and DBS 6, 7, 8. Clockwise (A) ICR1 dosed at $1 \mathrm{mg} / \mathrm{kg}$. (B) ICR2 dosed at $5 \mathrm{mg} / \mathrm{kg}$. (C) ICR3 dosed at $5 \mathrm{mg} / \mathrm{kg}$. (D) ICR4 dosed at $10 \mathrm{mg} / \mathrm{kg}$.

\begin{tabular}{|c|c|c|c|c|c|c|c|c|}
\hline \multirow{3}{*}{ ICR1 } & \multirow{2}{*}{\multicolumn{2}{|c|}{$\begin{array}{c}\text { AUC }_{\text {last }} \\
\left(\mathrm{h}^{*} \mathrm{nmol} / \mathrm{L}\right)\end{array}$}} & \multirow{2}{*}{\multicolumn{2}{|c|}{$\begin{array}{c}\mathrm{CL} \\
(\mathrm{ml} / \mathrm{min} / \mathrm{kg})\end{array}$}} & \multicolumn{2}{|c|}{ HL_Lambda_z } & \multirow{2}{*}{\multicolumn{2}{|c|}{$\begin{array}{c}\mathbf{V}_{\mathrm{ss}} \\
(\mathrm{L} / \mathrm{kg})\end{array}$}} \\
\hline & & & & & \multirow{2}{*}{$\begin{array}{l}\text { (h) } \\
\text { av. }\end{array}$} & \multirow[b]{2}{*}{ sd } & & \\
\hline & av. & sd & av. & sd & & & av. & sd \\
\hline CMS & 3200 & 117 & 9.14 & 0.46 & 2.87 & 0.57 & 2.08 & 0.32 \\
\hline DBS & 3087 & 28 & 9.79 & 0.64 & 2.61 & 0.46 & 2.04 & 0.22 \\
\hline \multirow{3}{*}{ ICR2 } & \multicolumn{2}{|c|}{ AUC $_{\text {last }}$} & \multicolumn{2}{|c|}{ CL } & \multicolumn{2}{|c|}{ HL_Lambda_z } & \multicolumn{2}{|c|}{$\mathbf{v}_{\mathrm{ss}}$} \\
\hline & \multicolumn{2}{|c|}{$\left(\mathrm{h}^{*} \mathrm{nmol} / \mathrm{L}\right)$} & \multicolumn{2}{|c|}{$(\mathrm{ml} / \mathrm{min} / \mathrm{kg})$} & \multicolumn{2}{|c|}{ (h) } & \multicolumn{2}{|c|}{$(\mathrm{L} / \mathrm{kg})$} \\
\hline & av. & sd & av. & sd & av. & sd & av. & sd \\
\hline CMS & 8190 & 1528 & 25.34 & 5.15 & 0.68 & 0.04 & 1.15 & 0.34 \\
\hline DBS & 6899 & 220 & 29.25 & 0.92 & 0.75 & 0.07 & 1.62 & 0.04 \\
\hline \multirow{3}{*}{ ICR3 } & \multirow{2}{*}{\multicolumn{2}{|c|}{$\begin{array}{c}\text { AUC }_{\text {last }} \\
\left(\mathrm{h}^{*} \mathrm{nmol} / \mathrm{L}\right)\end{array}$}} & \multicolumn{2}{|c|}{ CL } & \multicolumn{2}{|c|}{ HL_Lambda_z } & \multicolumn{2}{|c|}{$\mathrm{v}_{\mathrm{ss}}$} \\
\hline & & & \multicolumn{2}{|c|}{$(\mathrm{ml} / \mathrm{min} / \mathrm{kg})$} & \multicolumn{2}{|c|}{ (h) } & \multicolumn{2}{|c|}{$(\mathrm{L} / \mathrm{kg})$} \\
\hline & \multicolumn{2}{|l|}{ av. } & av. & sd & av. & sd & av. & sd \\
\hline CMS & 14420 & 2157 & 9.61 & 1.81 & 1.65 & 0.23 & 1.14 & 0.02 \\
\hline DBS & 13660 & 3974 & 10.13 & 2.66 & 1.87 & 0.20 & 1.50 & 0.42 \\
\hline \multirow{3}{*}{ ICR4 } & \multicolumn{2}{|c|}{ AUC $_{\text {last }}$} & \multicolumn{2}{|c|}{ CL } & \multicolumn{2}{|c|}{ HL_Lambda_z } & \multicolumn{2}{|c|}{$\mathrm{V}_{\mathrm{ss}}$} \\
\hline & \multicolumn{2}{|c|}{$\left(\mathrm{h}^{*} \mathrm{nmol} / \mathrm{L}\right)$} & \multicolumn{2}{|c|}{$(\mathrm{ml} / \mathrm{min} / \mathrm{kg})$} & \multicolumn{2}{|c|}{ (h) } & \multicolumn{2}{|c|}{$(\mathrm{L} / \mathrm{kg})$} \\
\hline & av. & sd & av. & sd & av. & sd & av. & sd \\
\hline CMS & 5406 & 240 & 88.46 & 4.94 & 1.15 & 0.19 & 6.29 & 0.57 \\
\hline DBS & 5678 & 972 & 85.64 & 15.42 & 1.35 & 0.21 & 5.81 & 1.21 \\
\hline
\end{tabular}

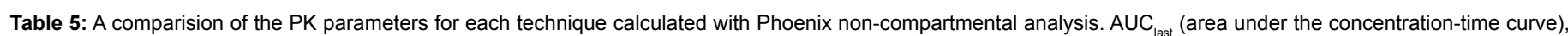
$\mathrm{CL}$ (clearance), HL_Lambda_z (half-life), $\mathrm{V}_{\text {ss }}$ (volume of disribution at steady state).

a $-80^{\circ} \mathrm{C}$ freezer, we have successfully carried out additional studies with other compounds where a more affordable $-20^{\circ} \mathrm{C}$ freezer was used. Extraction of both CMS and DBS requires plates and solvents and there is little financial difference in these consumables, rather the saving comes in the form of analyst time as CMS extraction takes less time than the manual punching of the DBS cards. 
Citation: Roberts J, Wilson I, Henley AT, Richards M, Thai C, et al. (2016) Capillary Microsampling of Mouse Blood in Early Pre-Clinical Studies: A Preferred Alternative to Dried Blood Spot Sampling. J Bioanal Biomed 8: 028-033. doi:10.4172/1948-593X.1000149

CMS can be readily integrated to workflows using liquid handling systems with little extra expense, unlike DBS where automated extraction is possible but requires extra instrumentation and/or addons to mass spectrometers. Capillary based studies also have the advantage of a choice of matrices; blood can be extracted directly or the capillaries can be centrifuged to yield plasma [13].

Using laboratory automation platforms increases costs compared to manual pipetting although this is balanced by an increase in consistency and precision. There is also the added benefit of speeding up the analytical workflow and reducing the risk of repetitive strain injuries through pipetting and punching cards.

Lowering the sample volume allows flexibility for multiple aliquots per time point offering a second analytical chance (providing Home Office guidelines and project licences are not exceeded). Separate samples taken at the same time point would enable analysis by two different methods, for example in PK/PD studies drug determination and biomarker estimation may require different detection techniques.

In conclusion, this study demonstrates that results from DBS and CMS protocols are comparable for novel and structurally differentiated chemical entities from four oncology drug discovery programmes in the ICR portfolios. CMS has great potential to replace DBS sampling in pre-clinical studies; it is easy to perform and readily integrates with automated liquid handling platforms with a much lower financial outlay compared to an automated DBS system. Replacing the manual DBS workflow with an automated CMS methodology offered a simple solution to streamline workflows between in vivo and bioanalytical laboratories. Refining the sampling technique by reducing sample volumes improved animal welfare by reducing restraint times.

\section{Acknowledgement}

This work was supported by a Cancer Research UK programme grant (C309/A11566) at the Cancer Research UK Cancer Therapeutics Unit. Thanks to colleagues from medicinal chemistry Professor lan Collins, Dr John Caldwell, Professor Keith Jones, Dr Elisa Pasqua, Professor Julian Blagg, Miss Aurelie Mallinger, Dr Swen Hoelder, Dr Hannah Woodward at the Cancer Research UK Cancer Therapeutics Unit and our collaborating partners for provision of the compounds evaluated in this work.

\section{References}

1. Tentler JJ, Choon Tan A, Weekes CD, Jimeno A, Leong S, et al. (2012) Patientderived tumour xenografts as models for oncology drug development. Nature Reviews Clinical Oncology 9: 338-350.

2. Day C, Merlino G, Van Dyke T (2015) Preclinical Mouse Cancer Models: A Maze of Opportunities and Challenges. Cell 163: 39-53.

3. Peng SX, Rockafellow BA, Skedzielewski TM, Huebert ND, Hageman W (2009) Improved Pharmacokinetic and Bioavailability Support of Drug Discovery Using Serial Blood Sampling in Mice. J Pharm Sci 98: 1877-1884.

4. National Centre for the Replacement Refinement \& Reduction of Animals in Research.

5. Kurawattimath V, Pocha K, Thanga Mariappan T, Trivedi RK, Mandlekar S (2012) A modified serial blood sampling technique and utility of dried-blood spot technique in estimation of blood concentration: application in mouse pharmacokinetics. Eur J Drug Metab Pharm 37: 23-30.

6. Beaudette P, Bateman KP (2004) Discovery stage pharmacokinetics using dried blood spots. J Chromatogr B 809: 153-158.

7. Li W, Tse FL (2010) Dried blood spot sampling in combination with LC - MS / MS for quantitative analysis of small molecules. Biomed Chromatogr 24: 49-65.

8. De Kesel PMM, Sadones N, Capiau S, Lambert WE, Stove CP (2013) Hemato - critical issues in quantitative analysis of dried blood spots: challenges and solutions. Bioanalysis 5: 2023-2041.

9. Jonsson O, Palma Villar P, Nilsson LB, Norsen-Höög C, Brogen J, et al. (2012) Capillary microsampling of $25 \mu \mathrm{l}$ blood for the determination of toxicokinetic parameters in regulatory studies in animals. Bioanalysis 4: 661-674.

10. Workman P, Aboagye EO, Balkwill F, Balmain A, Bruder G, et al. (2012) Guidelines for the Welfare and use of animals in cancer research. $\mathrm{Br} \mathrm{J}$ Cancer 102: $1555-1577$.

11. Timmerman P, White S, Cobb Z, Woods K, de Vries R, et al. (2013) Update of the EBF recommendation for the use of DBS in regulated bioanalysis integrating the conclusions from the EBF DBS-microsampling consortium. Bioanalysis 5: 2129-2136.

12. Timmerman P, White S, Cobb Z, Woods K, de Vries R, et al. (2014) European Bioanlaysis Forum continued plans to support liquid microsampling. Bioanalysis 6: 1897-1900.

13. Jonsson O, Palma Villar P, Nilsson LB, Eriksson M, Königsson K (2012) Validation of a bioanalytical method using capillary microsampling of $8 \mu \mathrm{l}$ plasma samples: application to a toxicokinetic study in mice. Bioanalysis 4 : 1989-1998. 